\title{
ARTICLE
}

\section{Understanding and managing cognitive impairment in bipolar disorder in older people}

\author{
Judy S. Rubinsztein (1), Barbara J. Sahakian \& John T. O'Brien
}

Judy S. Rubinsztein is a consultant in old age psychiatry at Fulbourn Hospital, Cambridge, and an associate lecturer at the University of Cambridge, UK. Her research interests focus on the neuropsychology, epidemiology and treatments of bipolar disorder in older people and in dementia. Barbara J. Sahakian is Professor of Clinical Neuropsychology at the University of Cambridge. She is a Fellow of the Academy of Medical Sciences. She has research interests across a wide range of neuropsychiatric conditions, including affective disorders, addiction, dementia, attention-deficit hyperactivity disorder and obsessive-compulsive disorder. Her research focuses on neuropsychology, neuropsychopharmacology, neuroimaging and neuroethics of these neuropsychiatric disorders.

John T. O'Brien is Professor of Old Age Psychiatry at the University of Cambridge. He is a National Institute for Health Research (NIHR) emeritus senior investigator and Fellow of the Academy of Medical Sciences. He has research interests in affective disorders in older people, in neuroimaging in dementia, Lewy body

dementia and clinical trials. His research focuses on the role of biomarkers such as MRI, SPECT and PET imaging and the role of vascular disease and inflammation in affective disorders in older people.

Correspondence Judy Rubinsztein, Beechcroft, Fulbourn Hospital,

Fulbourn, Cambridge CB21 5EF, UK. Email: judy.rubinsztein@cpft.nhs.uk

First received 10 May 2018 Final revision 13 Dec 2018 Accepted 14 Dec 2018

\section{Copyright and usage}

(C) The Royal College of Psychiatrists 2019

\section{SUMMARY}

Bipolar disorder is less prevalent in older people but accounts for $8-10 \%$ of psychiatric admissions. Treating and managing bipolar disorder in older people is challenging because of medical comorbidity. We review the cognitive problems observed in older people, explore why these are important and consider current treatment options. There are very few studies examining the cognitive profiles of older people with bipolar disorder and symptomatic depression and mania, and these show significant impairments in executive function. Most studies have focused on cognitive impairment in euthymic older people: as in euthymic adults of working age, significant impairments are observed in tests of attention, memory and executive function/processing speeds. Screening tests are not always helpful in euthymic older people as the impairment can be relatively subtle, and more in-depth neuropsychological testing may be needed to show impairments. Cognitive impairment may be more pronounced in older people with 'late-onset' bipolar disorder than in those with 'early-onset' disorder. Strategies to address symptomatic cognitive impairment in older people include assertive treatment of the mood disorder, minimising drugs that can adversely affect cognition, optimising physical healthcare and reducing relapse rates.

\section{LEARNING OBJECTIVES}

After reading this article you will be able to:

- understand that cognitive impairment in euthymic older people with bipolar disorder is similar to that in working-age adults with the disorder, affecting attention, memory and executive function/processing speeds

- recognise that cognitive impairment in older people is likely to be a major determinant of functional outcomes

- Implement approaches to treat cognitive impairment in bipolar disorder.

\section{DECLARATION OF INTEREST}

B.J.S. consults for Cambridge Cognition, PEAK (www.peak.net) and Mundipharma.

\section{KEYWORDS}

Cognitive neuroscience; comorbidity; bipolar affective disorders; out-patient treatment; inpatient treatment.
The fictitious vignette presented in Box 1 captures the complexity of treating older people with bipolar disorder. Mrs $\mathrm{R}$ had complex medical comorbidity, i.e. polymyalgia rheumatica and presumed steroid-induced mania, chronic kidney disease and a urinary tract infection with delirium. Although her mood was no longer overtly depressed towards the end of the admission, she was noted to have residual cognitive impairment and symptoms such as demotivation and loss of confidence, which affected her ability to cope with activities of daily living (ADLs) and delayed her return home.

\section{Epidemiology and prognosis of bipolar disorder in older people}

Bipolar disorder in older people (Box 2) has an estimated prevalence rate of $0.5-1 \%$, based on several epidemiological studies (Sajatovic 2015). However, a recent study in the UK (Marwaha 2016) showed an even lower prevalence, of $0.4 \%$ in those aged 64-74 years of age. None of the participants aged 75 years and over screened positive for bipolar disorder. The management and treatment of bipolar disorder remains a significant part of an old age psychiatrist's caseload and has been shown to account for about $8-10 \%$ of old age in-patient admissions across the world (Depp 2004).

Life expectancy in bipolar disorder is reduced, with premature death 1.77 times higher than the general population average (Hayes 2017). It has been demonstrated that life expectancy of a 15-year-old with bipolar disorder is reduced by 12.7 years for males and 8.9 years for females (Kessing 2015). Natural causes account for a large majority of premature deaths.

In older people with bipolar disorder medical comorbidities are increased. One review showed an average of 3-4 such comorbidities (Lala 2012). Another review showed that the dominant comorbid conditions in older adults with bipolar disorder were type 2 diabetes, respiratory and cardiovascular conditions and other endocrine abnormalities (Rise 2016). It is not certain whether the increased prevalence of comorbidity described in these studies is a consequence of treatment, lifestyle or directly 
attributable to the severe mental illness, but it is likely to be a combination of these factors.

\section{Cognitive impairment in bipolar disorder in older people}

Cognitive changes are important in bipolar disorder in all phases of the illness and in euthymia. In mania and hypomania poor concentration and attention, distractibility and the increased speed of mental activity are notable features in the ICD-10 and DSM-5 classifications (World Health Organization 1992; American Psychiatric Association 2013). Similarly, in depression reduced attention and concentration and slowness or retardation of thinking are recognised as part of the illness. The literature relating to cognitive changes in bipolar mania/ hypomania and depression in older people is sparse. Mixed affective disorders and rapid cycling presentations are frequently seen in older people and bring more 'chaos' to the clinical scenario and probably more cognitive impairment. However, there are no formal cognitive studies known to us examining cognition in such mixed states or in rapid cycling in older people. Most studies in older people have been conducted during the euthymic phase of bipolar disorder and these studies help us to understand the underlying 'cognitive syndrome' in bipolar disorder separate from the superadded cognitive impairment that may relate to specific mood changes.

The structure of the review we present here is outlined in Box 3.

\section{Cognitive impairment in different phases of bipolar disorder in older people}

\section{In mania}

We found few studies of symptomatic mania in older people. A small study $(n=14)$ confirmed significant impairments in participants with bipolar mania on executive tasks of initiation/perseveration and response inhibition compared with those with

\section{BOX 2 Some terminology explained}

- Older people (older adults) refers to people aged 65 years and above

- Working-age adults refers to people aged 17-64

- Early-onset versus late-onset bipolar disorder: studies vary in definitions, with late-onset illness describing a first affective episode at ages ranging from 40 to 60

BOX 1 Case vignette: Mrs R

Mrs $R$ is a 72-year-old widow with earlyonset bipolar disorder (she experienced a first affective episode at the age of 26). She has remained well on lithium for the past 10 years and has been looked after in primary care. She has coped with all activities of daily living, including shopping and cooking. She became hypomanic after receiving steroid treatment for polymyalgia rheumatica 3 months ago. Her mental state improved when the dose of steroids was reduced. Her general practitioner noted that her renal function was impaired (estimated glomerular filtration rate, eGFR, had declined from 52 to 40) and she was referred to a community psychiatrist in secondary care for advice on whether to stop the lithium.

Mrs R was euthymic when seen by the community psychiatrist. The pros and cons of stopping lithium and commencing sodium valproate were discussed with her. Unfortunately, soon after stopping lithium, Mrs $R$ became very depressed to the point of requiring a psychiatric hospital admission. Her food and fluid intake were poor, and she was unable to look after herself. Soon after the psychiatric admission she had to be transferred to an acute hospital for intravenous antibiotics. She returned to the psychiatric ward with evidence of a mild delirium. The delirium resolved in a week, but she remained very depressed. She refused electroconvulsive therapy (ECT), which had been helpful in the past. She was recommenced on a low dose of lithium (eGFR had returned to 50) and antidepressants. She made a slow recovery in hospital over several months and her mood improved to a euthymic state. She came off all the prednisolone slowly with the advice of a rheumatologist. She was able to socialise on the ward and participated in all therapeutic activities. However, she had lost confidence and motivation to cope with activities of daily living at home. Neuropsychological examination confirmed that she had cognitive impairment across attention and memory tasks (both scored 0.5 s.d. below norms), and on executive tasks she showed considerable difficulties with planning and switching (11.5 s.d. below norms). She needed assistance to return home and initially received help twice a day with cooking, shopping and housework. However, after a few months her confidence improved, and she no longer needed a care package.
BOX 3 Structure of this review on cognitive studies in older people with bipolar disorder

This review of the literature (from 2000 to June 2017) first focuses on what is known about symptomatic cognitive impairment in older people:

- during the manic phase

- during depressive episodes

- in the euthymic state

We then discuss why understanding cognitive impairment in bipolar disorder is important in older people in terms of:

- differentiating between cognitive impairment related to bipolar disorder itself and a newly acquired dementia syndrome

- recognising that dementia is more common in people with bipolar disorder than in the general population

- understanding functional outcome

- considering the question of neuroprogression

- examining current treatment options: drugs and psychological therapies. 
unipolar depression or control participants (Gunning-Dixon 2008). In a large sample of 87 patients aged 60 years and over in a manic state, $39 \%$ scored 1 s.d. or below on the Dementia Rating Scale (DRS), used as a measure of overall cognitive ability (Gildengers 2010). Further analysis of DRS scores showed that the percentage of participants scoring 1 or more s.d. below the age-adjusted means on specific domains were: conceptualisation, $31 \%$; memory, $31 \%$; initiation and perseveration, 20\%; construction, 17\%; and attention, $13 \%$. The more severe the mania, the lower the overall scores on the DRS. There was no correlation between lifetime duration of bipolar disorder and the overall DRS or subscale scores or vascular disease burden.

\section{In bipolar depression}

There are very few studies examining in detail the cognitive profile of older people with bipolar depression. One study examined executive function in this group (Caixeta 2017). On a battery of standard executive function tests, it demonstrated that at least one executive task was significantly impaired (<2 s.d. below norms) in $57 \%$ of patients. The study did not account for the severity of depressive symptoms or have a control group. Further research is needed to enable us to better understand, manage and treat such core cognitive problems in bipolar disorder in older people.

\section{In euthymia}

In the most comprehensive meta-analysis of cognitive impairment in older people with euthymic bipolar disorder, the authors (Samamé 2013) demonstrated the presence of significant cognitive impairment. This analysis helps us to understand the underlying 'cognitive syndrome' in euthymia as opposed to changes attributable to 'mood' in bipolar disorder. The authors showed that, across ten cognitive tests, overall effect sizes ranged from 0.61 to 0.88 on sustained attention, digit span (forwards and backwards), delayed recall, serial learning, cognitive flexibility and verbal fluency tasks. They concluded that, on average, cognitive performance of elderly patients with bipolar disorder was between 0.6 and 0.9 s.d. below that of healthy individuals and that these results were very similar to those reported in working-age adults. These cognitive changes are not often evident on screening tests such as the Mini-Mental State Examination (MMSE) and clock-drawing test, and a more sophisticated battery of tests is often needed to show cognitive impairment in this group. The use of more comprehensive bedside tests such as the Addenbrooke's Cognitive Examination (ACE) or Montreal Cognitive Assessment (MoCA) has not been evaluated in older people with bipolar disorder. Computerised neuropsychological batteries such as the Cambridge Neuropsychological Test Automated Battery (CANTAB) have been shown to be sensitive to impairments in euthymic working-age adults with bipolar disorder (Rubinsztein 2000).

Only a few studies examine the question of whether older people with 'late-onset' bipolar disorder are worse off cognitively than those with 'early-onset' illness. Definitions vary as to the cutoff age for defining late-onset versus early-onset bipolar disorder, which is not always helpful. Two studies that both define late onset as a first affective episode after 40 years of age, showed that older people with late-onset disorder showed more extensive and severe cognitive impairment than those with early-onset illness (Schouws 2009; Martino 2013). In the larger study (Schouws 2009), 59 individuals with early-onset bipolar disorder were compared with 60 individuals with late-onset illness and shown to have similar cerebrovascular risk factors. This study demonstrated that psychomotor performance, word fluency and mental flexibility were more impaired in the late-onset than the early-onset group. In a comparison between 10 older adults with late onset (defined in this study as onset after 60 years) and 49 with early-onset bipolar disorder using magnetic resonance imaging there was a greater prevalence of white matter hyperintensities in the late-onset group (Tamashiro 2008). This study needs replication in larger numbers, but it supports the hypothesis that vascular-related mechanisms may be more relevant in the aetiology of bipolar disorder in the late-onset group.

\section{Potential confounders in neuropsychological studies}

There may be other contributing factors to cognitive impairment in bipolar disorder, other than the 'illness' itself. Medication for bipolar disorder may contribute both positively and negatively to cognitive impairment. As the literature on older people is limited, we need to look to the literature on younger adults to examine the possible effects of medication on cognition. Some (Cullen 2016) show an association between the degree of cognitive impairment and treatment with antipsychotics but not lithium or anti-epileptics. Others (Bourne 2013) found cognitive impairments to be unrelated to drug treatments but acknowledge that their analysis did not take into account duration or dosage of drug treatments. An epidemiological study reported that lithium may provide neuroprotection against dementia in older people with bipolar disorder (Nunes 2007). However, some reviews conclude that lithium has a negative effect on memory 
and speed of information processing in bipolar disorder (Honig 1999). The number of studies included in Honig et al's analysis was small and methodology acknowledged to be weak. Against this is a study that shows that cognitive impairment persists in medication-free patients (Goswami 2009). Furthermore, in a longitudinal study of memory tests in patients taking lithium for bipolar disorder, test scores remained stable over the 6-year period, with only one of the ten subtests showing a statistical but not clinically significant decrease (Engelsmann 1988).

Another confounder for observing cognitive impairment is the potential presence of residual symptoms. More modern studies specifically try to exclude patients with significant residual symptoms by setting cut-offs on symptom ratings scales before inclusion. Studies in working-age adults examine for this in detail and demonstrate that residual symptoms cannot fully account for the effect sizes seen (Bourne 2013).

\section{Why is understanding symptomatic cognitive impairment in older adults important and how do we manage it?}

Why we need to understand it

Differentiating from dementia

First, older people and their clinicians may have heightened concerns about cognitive problems, and it is often difficult to differentiate cognitive impairment that is part of bipolar disorder itself from the onset of a newly acquired organic syndrome such as dementia.

In a person with bipolar disorder presenting with memory concerns it is helpful to explore whether baseline cognitive tests were done in the past when the patient was euthymic. Otherwise, clinicians may need to follow the patient longitudinally, as worsening cognition together with worsening functional abilities remain the cornerstone of a dementia diagnosis.

Some academic studies have compared older people with bipolar disorder with those with mild cognitive impairment to see whether there are clear differences in their cognitive profiles. These studies showed that euthymic patients with bipolar disorder performed similarly to patients with mild cognitive impairment (MCI) on tests of memory, executive functioning and processing speeds. In a smaller study (Silva 2009), patients with MCI showed fewer problems on an episodic memory test, but this was not replicated in the larger study (Osher 2011).

Sometimes the symptoms of bipolar disorder mimic those of behavioural-variant frontotemporal dementia (bvFTD). A small study comparing euthymic patients with bipolar disorder, patients with
bvFTD and controls showed that the bvFTD group demonstrated significantly greater impairments in executive function and theory of mind than the bipolar group (Baez 2017). Structural imaging changes in grey matter volumes were also markedly more atrophic in the bvFTD group compared with the bipolar group in frontal, temporal and parietal regions.

\section{Higher risk of dementia}

Second, dementia has been shown to be more common in people with a history of bipolar disorder than in the general population in several epidemiological studies (Diniz 2017). We know that cardiovascular disease, obesity and diabetes are often comorbid conditions in bipolar disorder and may contribute to both higher rates of vascular dementia and premature death. The onset of cognitive decline may therefore represent an early set of signs of comorbid dementia in bipolar disorder. This may be hard to unpick from the cognitive impairment related to the bipolar disorder itself.

\section{Effects on outcomes}

Third, cognitive impairment may be an important determinant of overall functional outcome in bipolar disorder. In working-age adults, functional recovery may happen in only a third of patients and cognitive impairment may persist in remission and will probably determine outcomes (Baune 2015). In older people, there are far fewer studies examining the relationship between euthymia and overall functioning, but these support the literature in younger people. Older patients with bipolar disorder in remission showed impairments in information processing speed and executive function that correlated with impaired instrumental activities of daily living (IADLs) (Gildengers 2007) and selfcare (IADLs and ADLs) (Pennarts 2014). Such functional impairments may be important determinants of whether patients will manage at home or are at risk of institutionalisation.

\section{Illuminating the neuroprogression debate}

Fourth, understanding cognitive impairment in older people may shed light on the debate as to whether bipolar disorder is a neuroprogressive disorder. Some authors have suggested that bipolar disorder is a neuroprogressive illness because of these associations between course of illness and cognitive impairment and the increased prevalence of dementia in bipolar disorder (Diniz 2017). Markers of illness severity vary across studies, but associations have been found between, for example, the number of manic episodes, the number of hospital admissions or illness episodes and the degree of cognitive 
BOX 4 Some notes on management of symptomatic cognitive impairment in older adults with bipolar disorder

- First treat mood disorder assertively

- Cognitive impairment should not preclude the use of electroconvulsive therapy (ECT) for bipolar depression

- Minimise the use of anticholinergic medications for comorbid physical illnesses

- There is currently no evidence in favour 'dementia drugs' - acetylcholinesterase inhibitors and memantine

- Functional remediation - strategies to tackle impairment in attention, memory and executive functioning - has shown some success in working-age adults with bipolar disorder

impairment. From these studies we may infer that it is likely to be important to reduce relapse rates to preserve cognitive function. Cross-sectional studies in older people with bipolar disorder do not particularly support the neuroprogressive hypothesis, as cognitive impairments in euthymic older adults are similar to those seen in euthymic working-age adults. In a 5-year follow-up study in working-age adults with bipolar disorder (Santos 2014) the speed of processing, working memory, attention, verbal and visual memory and executive function tasks were persistent and stable after 5 years, with only verbal recall worsening a little. However, the controversy deepens when examining structural imaging studies. Significant white matter hyperintensities are observed in both young and old patients with bipolar disorder (although the aetiology may differ) (Besga 2011). Cognitive function was not shown to relate primarily to increased white matter hyperintensities in older adults with bipolar disorder (Rej 2014). However, lower total grey matter volumes did relate to longer duration of bipolar disorder and to greater antipsychotic exposure (Gildengers 2014).

\section{How we might manage it}

As cognitive problems may be a core part of bipolar disorder, there is a need to treat the mood state assertively in older people as this may lead to improvements in cognition. In a patient with bipolar disorder the question of whether there is a newly acquired dementia should be addressed only after active management of the mood disorder. Treating bipolar depression in older people may be especially challenging as treatment options are limited in their effectiveness (Pacchiarotti 2013) and medical comorbidity may further restrict choices (Box 4).
Electroconvulsive therapy (ECT) can be an effective course of action, and the presence of cognitive impairment should not preclude its use. A study examining this issue found that baseline cognitive scores were not a predictor of ECT outcomes for elderly people with unipolar or bipolar depression (Bjolseth 2015).

Studies have shown an association between anticholinergic medications and confusion and dementia in older adults in general (Kalisch Ellett 2014), although not specifically in bipolar disorder. We therefore suggest that minimising drugs with anticholinergic side-effects is a prudent strategy for addressing symptomatic cognitive impairment.

\section{Acetylcholinesterase inhibitors and memantine}

In line with results for unipolar depression (Reynolds 2011), a recent review in mainly working-age adults shows that acetylcholinesterase inhibitors (AChEIs) are not helpful in reducing mood or psychotic symptoms in bipolar depression and mania (Veronese 2016). However, the review reports on only three randomised controlled trials (RCTs) of AChEIs. These studies had small sample sizes ( $n=30$ or fewer), few examined cognition and results were conflicting. Only one study in older people (mean age of 74, $n=12$; Gildengers 2008) is reported on, and this was an open label study of donepezil that showed non-significant effects on cognitive measures. The review also comments on two RCTs in working-age adults comparing memantine with placebo. The first was small $(n=12)$ and reported improved response and remission rates for memantine (although not on the mood rating scores). In a large RCT ( $n=157)$ of memantine for depression in bipolar II disorder no improvement in mood was noted with the drug. The trial did not report on memantine's effects on cognition. Further research examining specifically whether cognition is improved by these drugs in older people with bipolar disorder is needed.

\section{An inflammatory response?}

Some have suggested that inflammation may play a role in the aetiology of cognitive impairment in bipolar disorder (Rosenblat 2015). Elevated serum levels of interleukin-1 receptor antagonist (IL-1RA) have been reported in euthymic older people with bipolar disorder and these raised levels were associated with worse cognitive function (Lotrich 2013). No correlations between IL-1RA and white matter integrity were found. There were no co-occurring increases in other cytokines, such as IL-6, by brain-derived neurotrophic factor. (Lotrich 2013). Although a causal relationship has not been established with IL-1RA this association 
provides support for this aetiological theory and opens up potential new treatment avenues to pursue.

\section{Psychotherapy}

We can find no studies examining psychological treatments for cognitive problems in older adults with bipolar disorder. The literature from studies of working-age adults suggests that functional remediation may hold some promise. This therapy involves a course of 21 sessions focusing on strategies to tackle the main areas of cognitive impairment (i.e. attention, memory and executive functioning). The efficacy of this therapy was demonstrated in an RCT comparing functional remediation with psychoeducation (which aims to prevent relapse) and treatment as usual (Torrent 2013). However, independent replication of this result is needed and use in older patients with bipolar disorder has not been established.

\section{Conclusions}

In older people, cognitive impairment on executive function tasks in bipolar depression and across a range of tasks in mania have been demonstrated in a limited number of studies. Cross-sectional studies of euthymic older people with bipolar disorder show significant cognitive impairment compared with matched control groups. There are correlations between the degree of cognitive impairment and impairments of psychosocial functioning that are likely to hinder recovery and rehabilitation. Prescribed drugs may be contributing to some of these cognitive changes, such as reduced information processing speeds, but are unlikely to be the sole contributor to cognitive impairment. There are currently no drug treatments that have been shown in RCTs to improve cognitive impairment in bipolar disorder in older people. However, actively treating mood symptoms, minimising drugs that can adversely affect cognition, optimising physical healthcare and reducing relapse rates are worthwhile strategies to consider. Future research should address the management of cognitive problems both pharmacologically and psychologically in older patients with bipolar disorder.

\section{References}

American Psychiatric Association (2013) Diagnostic and Statistical Manual of Mental Disorders (5th edn) (DSM-5). American Psychiatric Publishing

Baez S, Pinasco C, Roca M, et al (2017) Brain structural correlates of executive and social cognition profiles in behavioral variant frontotemporal dementia and elderly bipolar disorder. Neuropsychologia, Feb 17: doi 10.1016/j.neuropsychologia.2017.02.012 [Epub ahead of print].

Baune BT, Mahli G (2015) A review on the impact of cognitive dysfunction on social, occupational, and general functional outcomes in bipolar disorder. Bipolar Disorders, 17: 41-55.
Besga A, Martinez-Cengotitatabengoa M, Gonazlez-Ortega I, et al (2011) The role of white matter in late onset bipolar disorder. Maturitas, 70 : $160-3$.

Bjolseth TM, Engedal K, Benth JS, et al (2015) Baseline cognitive function does not predict the treatment outcome of electroconvulsive therapy (ECT) in late-life depression. Journal of Affective Disorders, 185: 67-75.

Bourne C, Aydemir 0, Blanza-Martinex V, et al (2013) Neuropsychological testing of cognitive impairment in euthymic bipolar disorder: an individual patient data meta-analysis. Acta Psychiatrica Scandinavica, 128: 149-62.

Caixeta L, Soares V, Vieira RT, et al (2017) Executive function is selectively impaired in old age bipolar depression. Frontiers in Psychology, 8: 194.

Cullen B, Ward J, Graham NA, et al (2016) Prevalence and correlates of cognitive impairment in euthymic adults with bipolar disorder: a systematic review. Journal of Affective Disorders, 205: 165-81.

Depp CA, Jeste DV (2004) Bipolar disorder in older adults: a critical review. Bipolar Disorder, 6: 343-67.

Diniz BS, Teixeira AL, Cao F, et al (2017) History of bipolar disorder and the risk of dementia: a systematic review and meta-analysis. American Journal of Geriatric Psychiatry, 25: 357-62.

Engelsmann F, Katz J, Ghadirian AM, et al (1988) Lithium and memory: a long-term follow-up study. Journal of Clinical Psychopharmacology, 8: 207-12.

Gildengers AG, Butters MA, Chisholm D, et al (2007) Cognitive functioning and instrumental activities of daily living in late-life bipolar disorder. Americal Journal of Geriatric Psychiatry, 15: 174-9.

Gildengers AG, Butters MA, Chisholm D, et al (2008) A 12 week openlabel pilot study of donepezil for cognitive functioning and instrumental activities of daily living in late-life bipolar disorder. International Journal of Geriatric Psychiatry, 23: 693-8.

Gildengers AG, Benoit $H$, Mulsant MD, et al (2010) The relationship of bipolar disorder lifetime duration and vascular burden to cognition in older adults. Bipolar Disorders, 12: 851-8.

Gildengers AG, Chung KH, Huand SH, et al (2014) Neuroprogressive effects of lifetime illness duration in older adults with bipolar disorder. Bipolar Disorders, 16: 617-23.

Goswami U, Sharma A, Varma A, et al (2009) The neurocognitive performance of drug-free and medicated euthymic bipolar patients do not differ. Acta Psychiatrica Scandinavica, 120: 456-63.

Gunning-Dixon FM, Murphy CF, Alexopoulos GS, et al (2008) Executive dysfunction in elderly bipolar manic patients. American Journal of Geriatric Psychiatry, 16: 506-12.

Hayes JF, Marston L, Walters K, et al (2017) Mortality gap for people with bipolar disorder and schizophrenia: UK-based cohort study 2000-2014. British Journal of Psychiatry, 211: 175-81.

Honig A, Arts BMG, Ponds RWMH, et al (1999) Lithium induced cognitive side-effects in bipolar disorder: a qualitative analysis and implications for daily practice. International Clinical Psychopharmacology, 14: 167-71.

Kalisch Ellett LM, Pratt NL, Ramsay EN, et al (2014) Multiple anticholinergic medication use and risk of hospital admission for confusion and dementia. Journal of the American Geriatric Society, 62: 1916-22.

Kessing LV, Vradi E, Andersen PK (2015) Life expectancy in bipolar disorder. Bipolar Disorders, 17: 543-8.

Lala SV, Sajatovic M (2012) Medical and psychiatric comorbidities among elderly individuals with bipolar disorder: a literature review. Journal of Geriatric Psychiatry and Neurology, 25: 20-5.

Lotrich FE, Butters MA, Aizenstein H, et al (2013) The relationship between interleukin-1 receptor antagonist and cognitive function in older adults with bipolar disorder. International Journal of Geriatric Psychiatry, 29: 635-44.

Martino DJ, Strejilevich SA, Manes F (2013) Neurocognitive functioning in early-onset and late-onset older patients with euthymic bipolar disorder. International Journal of Geriatric Psychiatry, 28: 142-8.

Marwaha S, Sal N, Bebbington P(2016) Bipolar disorder. In Mental Health and Wellbeing in England: Adult Psychiatric Morbidity Survey 2014 (eds S McManus, P Bebbington, R Jenkins, et al): 220-37. NHS Digital. 
Nunes PV, Forlenza OV, Gattaz WF (2007) Lithium and risk for Alzheimer's disease in elderly patients with bipolar disorder. British Journal of Psychiatry, 190: 359-60.

Osher Y, Dobron A, Belmaker RH, et al (2011) Computerized testing of neurocognitive function in euthymic bipolar patients compared to those with mild cognitive impairment and cognitively healthy controls. Psychotherapy and Psychosomatics, 80: 298-303.

Pacchiarotti I, Bond DJ, Baldesarini RJ, et al (2013) The International Society for Bipolar Disorders (ISBD) task force report on antidepressant use in bipolar disorders. American Journal of Psychiatry, 170: 1249-62.

Pennarts HM, Schouws SNTM, Bongers IMB (2014) Cognitive functioning in relation to self-care in elderly persons with a bipolar disorder. Clinical Gerontologist, 37: 419-28

Rej S, Butters MA, Aizenstein HJ, et al (2014) Neuroimaging and neurocognitive abnormalities associated with bipolar disorder in old age. International Journal of Geriatric Psychiatry, 29: 421-7.

Reynolds CF III, Butterss MA, Lopez 0, et al (2011) Maintenance treatment of depression in old age: a randomized, double-blind, placebo-controlled evaluation of the efficacy and safety of donepezil combined with antidepressant pharmacotherapy. Archives of General Psychiatry, 68: 51-60.

Rise IV, Haro J, Gjervan B (2016) Clinical features, comorbidity, and cognitive impairment in elderly bipolar patients. Neuropsychiatric Disease and Treatment, 12: 1203-13.

Rosenblat JD, Brietzke E, Mansur NA, et al (2015) Inflammation as a neurobiological substrate of cognitive impairment in bipolar disorder: evidence, pathophysiology and treatment implications. Journal of Affective Disorders, 188: 149-59.

Rubinsztein JS, Michael A, Paykel ES, et al (2000) Cognitive impairment in remission in bipolar affective disorder. Psychological Medicine, 30 : 1025-36.
Sajatovic M, Strejilevich SA, Gildengers A, et al (2015) A report on olderage bipolar disorder from the International Society for Bipolar Disorders Task Force. Bipolar Disorders, 17: 689-704.

Samamé C, Martino DJ, Strejilevich SA (2013) A quantitative review of neurocognition in euthymic late-life bipolar disorder. Bipolar Disorders, 15: 633-44.

Santos JL, Aparicio A, Bagney A, et al (2014) A five-year follow-up study of neurocognitive functioning in bipolar disorder. Bipolar Disorders, 16 722-31.

Schouws SNTM, Comijs HC, Stek ML, et al (2009) Cognitive impairment in early and late bipolar disorder. American Journal of Geriatric Psychiatry, 17: $508-15$

Silva D, Santana I, Simoes do Couto F, et al (2009) Cognitive deficits in middle-aged and older adults with bipolar disorder and cognitive complaints: comparison with mild cognitive impairment. International Journal of Geriatric Psychiatry, 24: 624-31.

Tamashiro JH, Zung S, Zanetti MV, et al (2008) Increased rates of white matter hyperintensities in late-onset bipolar disorder. Bipolar Disorders, 10: 765-75

Torrent C, Bonnin Cdel M, Martinez-Aran A et al (2013) Efficacy of functional remediation in bipolar disorder: a multicenter randomized controlled study. American Journal of Psychiatry, 170: 852-9.

Veronese N, Solmi M, Luchini C, et al (2016) Acetylcholinesterase inhibitors and memantine in bipolar disorder: a systematic review and best evidence synthesis of the efficacy and safety for multiple disease dimensions. Journal of Affective Disorders, 197: 268-80.

World Health Organization (1992) The ICD-10 Classification of Mental and Behavioural Disorders: Clinical Descriptions and Diagnostic Guidelines. WHO

\section{MCQs}

Select the single best option for each question stem

\section{Bipolar disorder has a prevalence in older people of around:}

a $10 \%$

b $0.5 \%$

c $5 \%$

d $2 \%$

e $20 \%$.

2 Common comorbid medical conditions in older people with bipolar disorder are:

a type 2 diabetes

b respiratory diseases

c cardiovascular conditions d endocrine abnormalities

e all of the above.

3 Cognitive impairment in euthymic patients with bipolar disorder may be subtle and may not be detected with:

a the Mini-Mental State Examination

b the clock-drawing test

c the Abbreviated Mental Test

$\mathrm{d}$ bedside questions

e all of the above.

4 Euthymic older patients with early-onset bipolar disorder have cognitive profiles:

a similar to younger patients

b much worse than younger patients c much better than younger patients

$d$ that deteriorate progressively every year

e that are none of the above.

5 Cognitive impairment in older people with bipolar disorder has been proven to improve significantly with:

a memantine

b donepezil

c rivastigmine

d risperidone

e none of the above. 